\title{
Relationship between Force Fluctuation in the Plantar Flexor and Sustainable Time for Single-leg Standing
}

\author{
Kazushige Oshita ${ }^{1), 2)}$ and Sumio Yano ${ }^{1)}$ \\ 1) Graduate School of Human Development and Environment, Division of \\ Human Environmental Science, Kobe University \\ 2) Japan Society for the Promotion of Science, Research Fellow DC
}

\begin{abstract}
The purpose of this study was to investigate the relationships between force fluctuation during isometric plantar flexion and the sustainable time for single-leg standing. Fourteen healthy males ( $21 \pm 1$ years) performed unilateral (preferred leg) force matching tasks and single-leg quiet standing. Force matching tasks were performed to maintain isometric plantar flexion for $15 \mathrm{~s}$ at levels corresponding to $10 \%$ and $20 \%$ maximal voluntary contraction (MVC) with the visual feedback of force. Force fluctuation during force matching tasks was quantified as the standard deviation of force. Sustainable time for single-leg quiet standing was performed to maintain a single-leg quiet standing barefoot on a platform using the preferred leg with their eyes closed. Force fluctuation was significantly greater in 20\% MVC task compared to $10 \% \mathrm{MVC}$ task. The sustainable time for singleleg quiet standing was strongly correlated with force fluctuation in $20 \%$ MVC task $(r=-0.56, p=0.04)$. However, it was not related to force fluctuation in $10 \%$ MVC task $(r=0.19$, $p=0.52)$ or MVC value $(\mathrm{r}=0.13, p=0.65)$. These results suggest that a specificity of contraction intensity is observed between force steadiness and the posture stability during single-leg quiet standing; force steadiness during 20\% MVC plantar flexion is one of the important components for posture stability during single-leg quiet standing. J Physiol Anthropol 29(3): 89-93, 2010 http://www.jstage.jst.go.jp/browse/jpa2
\end{abstract}

[DOI: $10.2114 /$ jpa2.29.89]

Keywords: force fluctuation, force steadiness, single-leg quiet standing, posture stability

\section{Introduction}

Human movement is caused by joint torque or muscle force generated by the contraction of multiple muscles. Force generated during voluntary muscle contraction is not constant but fluctuates. Fluctuations in muscle force during voluntary contractions can produce variability in movement, and this fluctuation is referred as the "force fluctuation." Force fluctuation is often influenced by multiple factors, including force level (Kouzaki et al., 2004; Shinohara et al., 2003; Oshita and Yano, 2010), fatigue (Hunter and Enoka, 2003; Maluf and Enoka, 2005), and inactivity (Shinohara et al., 2003), in normal young individuals. Hence, greater force fluctuation during muscle tasks can impair the performance of human movement. In the upper limbs, Kornatz et al. (2005) reported that a reduction in fluctuation of a hand muscle after training improves manual function as measured by the Purdue pegboard test (a manual dexterity test) in older adults. Further, Salonikidis et al. (2009) have also reported on young adults; highly skilled individuals present a greater ability to perform steady isometric contraction than less skilled participants. Therefore, force fluctuations can influence the functional ability of an individual to control the finger and limb movements during daily life.

The sustainable time for single-leg quiet standing with eyes open or closed is one of the classical posture stability tests, and performance in this test deteriorates with age (Bohannon et al., 1984; Haga et al., 1986; Rikli and Busch, 1986), fall (Haga et al., 1986), or a decrease in physical activity level (Rikli and Busch, 1986). This test is also established as one of the posture stability tests by the Japanese Ministry of Education, Culture, Sports, Science and Technology. Based on the dynamics of the human quiet stance, it has been observed that the plantar flexor muscles play a significant role in stabilizing the body during bipedal quiet stance (Masani et al., 2003; Morasso and Schieppati, 1999). Furthermore, the activities of the plantar flexor during bipedal quiet stance have been found to be coherent with both spontaneous body sway (Gatev et al., 1999; Masani et al., 2003) and mechanically induced body sway (Fitzpatrick et al., 1996). Although many factors (i.e., function of proprioceptor control of upper body motion, etc.) relate to posture stability during single-leg standing (especially, with eyes closed), the plantar flexor (the soleus muscle) indicate the highest muscle activates in the whole body muscles during single-leg quiet standing (Sawai et al., 2004). This literature 
led us to hypothesize that single-leg standing is associated with force fluctuation in the plantar flexor. If force fluctuation in the plantar flexor is one of the important factors for posture stability during single-leg quiet standing, the amplitude of the force fluctuation will indicate the relationship with ability with regard to posture stability. However, to the best of our knowledge, the relationship between force fluctuation in the plantar flexor and posture stability during single-leg standing is not currently known. Thus, the purpose of this study was to investigate the relationships between force steadiness in the plantar flexor and the sustainable time of single-leg quiet standing.

\section{Methods}

\section{Subject}

Data were obtained from fourteen healthy males $(21 \pm 1$ years). All subjects reported an absence of current or medical history of neuromuscular disorder. The subjects were informed of the purpose of the present study beforehand and a statement of informed consent was obtained. Further, this study had been approved by Human Ethics Committee of the Graduate School of Human Development and Environment, Kobe University.

\section{Setup}

Subjects performed a static unilateral plantar flexion exercise using the preferred leg. The preferred leg was determined by asking the participant which leg they believed was stronger and that they would kick a ball with (Bohannon et al., 1984). The preferred leg was the right leg in twelve subjects and the left leg in the other two subjects. The asymmetry of force fluctuation between legs was not observed during less than 20\% MVC contraction in lower limbs (Oshita and Yano, 2010). Subjects were seated on an insulated, straight-back chair. An additional strap was used to secure the thigh of the preferred leg to the chair. Force was measured with a strain-gauge transducer (LPR-A-S10, Kyowa, Tokyo, Japan) positioned between a metal base plate and the foot. The foot was secured with a strap at the foot lever plate. The straingauge transducer was aligned between the two plates near the distal part of the foot. The exact position of the entire device was carefully adjusted so that the knee was fully extended with the ankle joint angle at $90^{\circ}$. The produced force and the target were displayed on a PC monitor (14.1 inch) $1 \mathrm{~m}$ front of the subject to provide visual feedback.

\section{Maximal voluntary contraction (MVC)}

Subjects performed maximal voluntary plantar flexion for a period of $5 \mathrm{~s}$ with encouragement from the investigators. Each subject performed three trials, with subsequent trials performed if the differences in the peak force of two MVCs were $>5 \%$. Subjects were allowed to reject any effort that they did not regard as "maximal." The trial with the highest peak force was chosen for analysis.

\section{Force matching task}

On the basis of MVC measurement, the subject performed a steady isometric plantar flexion tasks for $15 \mathrm{~s}$ at a level corresponding to $10 \%$ and $20 \%$ of MVC. This target force was determined by the previous report (Sawai et al., 2004) that the intensity of the plantar flexor (the soleus muscle) during single-leg standing was below $20 \%$ MVC. The data were collected for one trial with each target, and the order of the target force was randomized across subjects. There was a rest period of $>1 \mathrm{~min}$ between trials and rest periods were also allowed up to five minutes between trials at the subject's request.

\section{Sustainable time for single-leg quiet standing}

Based on the method of Bohannon et al. (1984), subjects performed single-leg quiet standing with their eyes closed. Subjects were required to maintain a single-leg quiet stand barefoot on a platform on their preferred leg, with their arms held by their sides. The goal of each activity was to balance for $120 \mathrm{~s}$. In this test, three attempts were permitted for each subject. If the subject reached this goal, we recorded a time of $120 \mathrm{~s}$. If the subject did not reach his goal, we recorded the best of the three timed trials. There was a rest period of $>1$ min between trials and the rest periods were also allowed up to five minutes between trials at the subject's request.

\section{Date analysis}

The force signals were stored at a sample frequency of 1 $\mathrm{kHz}$ by sensor interface with a 24-bit analog-to-digital converter (PCD-300A, Kyowa, Tokyo, Japan) and stored on the hard disk of a personal computer. In the stored signals, the middle approximately $8 \mathrm{~s}$ (8192 sample) of the task was used for further analysis because there was no systematic change in fluctuations within trials. These data were processed with waveform analysis software SPCANA (ver 4.71, Japan) and Microsoft EXCEL. After band-pass filtering $(<100 \mathrm{~Hz})$, the standard deviation (SD) of the amplitude was calculated.

\section{Statistical analyses}

Linear regression analysis was conducted between the sustainable time for single-leg quiet standing and the other measured parameter (force fluctuations during task and MVC value). A paired t-test was used for comparison between the intensities of the changes in force fluctuation. These analyses were performed with JSTAT (version 12.5, Japan) software. In these data, significance was accepted to be at the $p<0.05$ levels. Data are presented as mean \pm standard error of the mean (SEM) unless otherwise stated.

\section{Results}

Mean sustainable time for single-leg quiet standing was $56.43 \pm 6.136 \mathrm{~s}$, and mean MVC was $275.4 \pm 40.3 \mathrm{~N}$. Figure 1 shows an example of force signals during plantar flexion in the $10 \%$ and $20 \%$ MVC tasks. Force fluctuation was significantly 


\section{$10 \%$ MVC}

\section{$20 \%$ MVC}

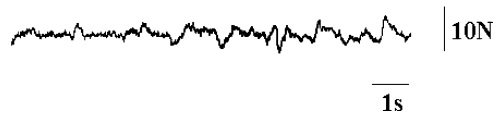

Fig. 1 An example of force signals during plantar flexion at $10 \%$ and $20 \%$ MVC.

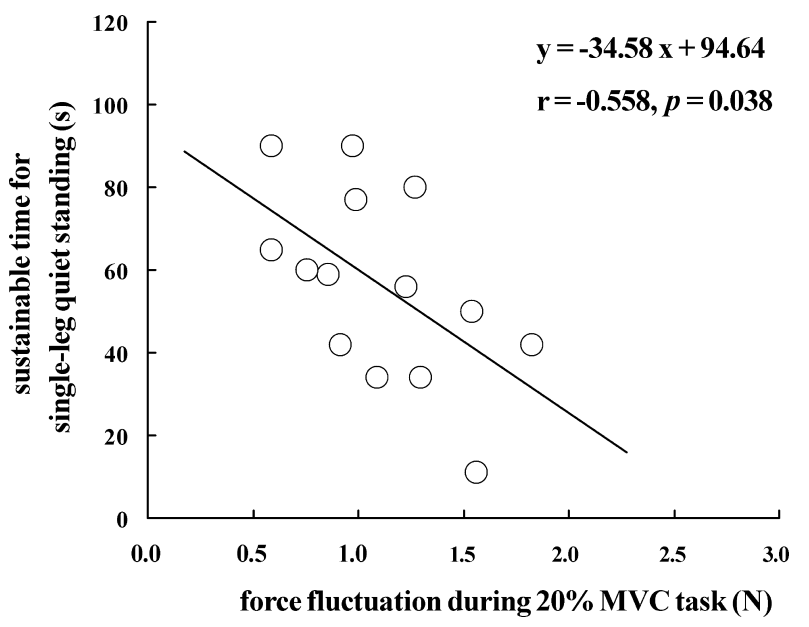

Fig. 2 Relationships between sustainable time for single-leg quiet standing and force fluctuation (quantified as the standard deviation (SD)) during isometric plantar flexion at 20\% MVC.

Sustainable time for single-leg quiet standing was strongly correlated with force fluctuation during isometric plantar flexion at $20 \% \mathrm{MVC}$ $(\mathrm{r}=-0.558, p=0.038)$.

greater in the $20 \%$ MVC task $(1.105 \pm 0.099 \mathrm{~N})$ compared to the $10 \%$ MVC task $(0.802 \pm 0.052 \mathrm{~N})(p=0.009)$. This result indicates that force fluctuation increase with contraction intensity.

Figure 2 shows the relationship between the sustainable time for single-leg quiet standing and force fluctuation in the $20 \%$ MVC task. Sustainable time for single-leg quiet standing was strongly correlated with force fluctuation in the $20 \%$ MVC task ( $\mathrm{r}=-0.558, p=0.038)$ (Fig. 2). Figure 3 shows the relationship between the sustainable time for single-leg quiet standing and force fluctuation in the 10\% MVC task. Although sustainable time for single-leg quiet standing was correlated with force fluctuation in the $20 \%$ MVC task, it was not related to force fluctuation in the $10 \%$ MVC task $(\mathrm{r}=0.189$, $p=0.518$ ) (Fig. 3). Furthermore, it was also not related to the MVC value $(\mathrm{r}=0.134, p=0.647)$. These results indicate that force steadiness during isometric contraction at $20 \% \mathrm{MVC}$ in the plantar flexor is an important factor for posture stability during single-leg quiet standing.

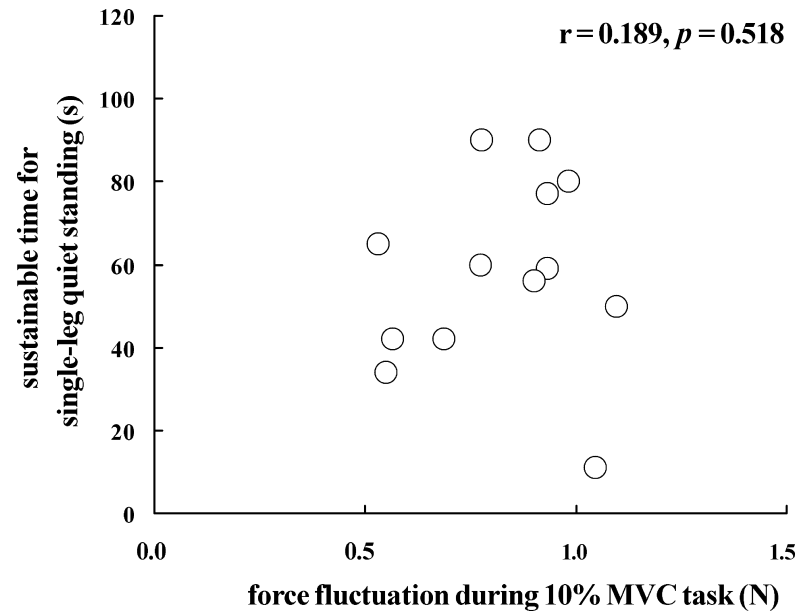

Fig. 3 Relationships between sustainable time for single-leg quiet standing and force fluctuation (quantified as the standard deviation (SD)) during isometric plantar flexion at $10 \%$ MVC.

Although sustainable time for single-leg quiet standing was strongly correlated with force fluctuation during isometric plantar flexion at $20 \%$ MVC (Fig. 2), it was not related to force fluctuation during isometric plantar flexion at $10 \% \mathrm{MVC}(\mathrm{r}=0.189, p=0.518)$.

\section{Discussion}

The main findings of this study were: (1) the force fluctuation during isometric plantar flexion was significantly increased in $20 \%$ MVC compared to $10 \%$ MVC; (2) the sustainable time for single-leg quiet standing was strongly correlated with the force steadiness during plantar flexion at $20 \%$ MVC; however, (3), it was not related to force steadiness during plantar flexion at $10 \% \mathrm{MVC}$ or the MVC value in the plantar flexor.

Several researchers have reported that force fluctuation increases with contraction intensity (Kouzaki et al., 2004; Shinohara et al., 2003; Oshita and Yano, in press), which is consistent with the results of the present study. Major determinants of the force fluctuation are thought to be the MU discharge rate variability or contractile property in active muscle (Mottram et al., 2005; Moritz et al., 2005; Tracy et al., 2005; Oshita and Yano, 2010). For example, previous studies revealed that force fluctuations indicate a positive correlation with the discharge rate variability during isometric contraction (Mottram et al., 2005; Tracy et al., 2005). Although the present study did not measure the discharge rate variability, force fluctuation was significantly increased with contraction intensity; which is consistent with the previous studies (Kouzaki et al., 2004; Shinohara et al., 2003; Oshita and Yano, 2010).

Force fluctuations during muscle tasks can influence the performance of human movement. Kornatz et al. (2005) reported that a reduction in fluctuation of a hand muscle after training improves manual function as measured by the Purdue pegboard test (a manual dexterity test) in older adults. Further, Salonikidis et al. (2009) have also reported on young adults; 
highly skilled individuals present a greater ability to perform steady isometric contraction than less skilled participants. Hence, force fluctuations can influence the functional ability of an individual to control the finger, hand, or limb during daily life. Further, the present result revealed that posture stability during single-leg quiet standing was strongly correlated with force steadiness during 20\% MVC plantar flexion. Sustainable time for single-leg quiet standing is one of the classical balance tests, and it deteriorates with age, fall, or inactivity (Bohannon et al., 1984; Haga et al., 1986; Rikli and Busch, 1986). For example, Haga et al. (1986) demonstrated that the sustainable time for single-leg quiet standing decreases with age, and that the experience of a fall was significantly decreased by the sustainable time for single-leg quiet standing in each age group. Rikli and Busch (1986) have also reported that Age $\times$ Activity level interaction was significant for the sustainable time for single-leg quiet standing. Further, the present result suggested that force steadiness during $20 \%$ MVC plantar flexion is one of the important factors for the ability of posture stabilization during single-leg quiet standing.

During bipedal quiet standing, the plantar flexor muscles play a significant role in stabilizing the body (Fitzpatrick et al., 1996; Masani et al., 2003; Morasso and Schieppati, 1999). The activities of the plantar flexor during bipedal quiet stance in both legs have been found to be coherent with both spontaneous body sway (Gatev et al., 1999; Masani et al., 2003) and mechanically induced body sway (Fitzpatrick et al., 1996). Further, the plantar flexor (the soleus muscle) is also indicated the highest muscle activates in the whole body muscles during single-leg quiet standing. This literature indicates that the function of the plantar flexor is one of the important factors for the ability of posture stability during single-leg quiet standing, and it is consistent with the present result: the force steadiness during $20 \%$ MVC plantar flexion was related to posture stability during single-leg quiet standing. Although the sustainable time for single-leg quiet standing was strongly correlated with force steadiness during $20 \%$ MVC plantar flexion, it was not related to force steadiness during $10 \%$ MVC plantar flexion or MVC value in the plantar flexor. Sawai et al. (2004) reported that the muscular activity level of the soleus muscle corresponded to about 20\% MVC during single-leg quiet standing. This previous study and our results indicate that the force steadiness during $20 \%$ MVC plantar flexion is appropriate to the ability of posture stability during single-leg quiet standing. Further, it is also suggested that $10 \% \mathrm{MVC}$ or MVC are too low or high intensity, respectively. This result suggests that a specificity of contraction intensity exists between force fluctuation and posture stability. Thus, if force steadiness in the plantar flexor applied to practice for improvement to posture stability (such as single-leg quiet standing), the target force have to establish for $20 \%$ MVC.

However, this study compared only sustainable time for single-leg quiet standing and force fluctuation. Therefore, future examination is required to measure the relationship between minutely kinematic or physiological parameters (i.e., center of mass displacement or electromyography analysis) and force steadiness. Furthermore, we have to consider the effect of visuomotor contribution. In recent years, Tracy (2007) reported that force fluctuation during visual feedback is greater than that of nonvisual feedback. In the present study, the force matching task was with visual feedback task whereas singleleg quiet standing was a nonvisual feedback task. However, when performing actual force steadiness practice, the participant will not know the exact target force in nonvisual feedback. The present study indicated that contraction intensity (target force) is an important factor for posture stability. Further, force steadiness during 20\% MVC plantar flexion with visual feedback was strongly correlated with posture stability during single-leg quiet standing without visual feedback. Therefore, force steadiness during $20 \%$ MVC plantar flexion is important for posture stability (such as single-leg quiet standing), even though the force steadiness is a visual feedback task.

These results will also provide useful information to design a training program for posture stability. Usually, the goal of many training programs is improvement of posture stability by an increase in muscle strength (Anderson and Behm, 2005; Holviala et al., 2006). Certainly, strength of the main working muscles to support self body weight is thought to be the most important factor for posture stability. However, MVC in the plantar flexor did not relate with posture sway in the present study. Further, Kouzaki et al. (2007) reported that postural sway during bipedal quiet standing increases following bed rest despite maintenance of the muscle volume of the main working muscle for human postural standing by strength training. These results indicate that not only muscle strength but also force steadiness is an important factor for posture stability.

In conclusion, the present study indicated that the sustainable time for single-leg quiet standing was strongly correlated with force steadiness during 20\% MVC plantar flexion. However, it was not related to force steadiness during $10 \%$ MVC plantar flexion or MVC in the plantar flexor. These results suggest that a specificity of contraction intensity exists between force steadiness and posture stability during single-leg standing; force steadiness during plantar flexion at $20 \%$ MVC is one of the important components for posture stability during single-leg standing.

Acknowledgement This work was supported by KAKENHI (Grant-in-Aid-for JSPS Fellows (21-2787) and Grant-in-Aid for Scientific Research "B" (20300235)).

\section{References}

Anderson K, Behm DG (2005) The impact of instability resistance training on balance and stability. Sports Med 35: 43-53

Bohannon RW, Larkin PA, Cook AC, Gear J, Singer J (1984) 
Decrease in Timed Balance Test Scores with Aging. Phys Ther 64: 1067-1070

ChristouEA, Jakobi JM, CritchlowA, FleshnerM, EnokaRM (2004) The 1- to $2-\mathrm{Hz}$ oscillations in muscle force are exacerbated by stress, especially in older adults. J Appl Physiol 97: 225-235

Fitzpatrick R, Burke D, Gandevia SC (1996) Loop gain of reflexes controlling human standing measured with the use of postural and vestibular disturbances. J Neurophysiol 76: 3994-4008

Gatev P, Thomas S, Kepple T, Hallett M (1999) Feedforward ankle strategy of balance during quiet stance in adults. J Physiol 514: 915-928

Haga H, Shibata H, Shichida K, Matsuzaki T, Hatano S (1986) Falls in the institutionalized elderly in Japan. Arch Gerontol Geriat 5: 1-9

Harris CM, Wolpert DM (1998) Signal-dependent noise determines motor planning. Nature 394: 780-784

Holviala JH, Sallinen JM, Kraemer WJ, Alen MJ, Häkkinen KK (2006) Effects of strength training on muscle strength characteristics, functional capabilities, and balance in middle-aged and older women. J Strength Cond Res 20: 336-344

Hunter SK, Enoka RM (2003) Changes in muscle activation can prolong the endurance time of a submaximal isometric contraction in humans. J Appl Physiol 94: 108-118

Kornatz KW, Christou EA, Enoka RM (2005) Practice reduces motor unit discharge variability in a hand muscle and improves manual dexterity in old adults. J Appl Physiol 98: 2072-2080

Kouzaki M, Shinohara M, Masani K, Fukunaga T (2004) Force fluctuations are modulated by alternate muscle activity of knee extensor synergists during low-level sustained contraction. J Appl Physiol 97: 2121-2131

Kouzaki M, Masani K, Akima H, Shirasawa H, Fukuoka H, Kanehisa H, Fukunaga T (2007) Effects of 20-day bed rest with and without strength training on postural sway during quiet stance. Acta Physiol 189: 279-292

Maluf KS, Enoka RM (2005) Task failure during fatiguing contractions performed by humans. J Appl Physiol 99: 389-396

Masani K, PopovicMR, Nakazawa K, KouzakiM, Nozaki D
(2003) Importance of body sway velocity information in controlling ankle extensor activities during quiet stance. J Neurophysiol 90: 3774-3782

Morasso PG, Schieppati M (1999) Can muscle stiffness alone stabilize upright standing? J Neurophysiol 82: 1622-1626

Mottram CJ, Christou EA, Meyer FG, Emoka RM (2005) Frequency modulation of motor unit discharge has taskdependent effects on fluctuations in motor output. J Neurophysiol 94: 2878-2887

Oshita K, Yano S (2010) Asymmetry of force fluctuation in knee extensor. Int J Sports Med 31: 342-346

Rikli R, Busch S (1986) Motor Performance of Women as a Function of Age and Physical Activity Level. J Gerontol 41: 645-649

Salonikidis K, Amiridis IG, Oxyzoglou N, de Villareal ES, Zafeiridis A, Kellis E (2009) Force variability during isometric wrist flexion in highly skilled and sedentary individuals. Eur J Appl Physiol 107: 715-722

Sawai S, Sanematsu H, Kanehisa H, Tsunoda N, Fukunaga T (2004) Evaluation of muscle activity level in daily actions. Jpn J Phys Fitness Sports Med 53: 93-106 [In Japanese with English Abstract]

Shinohara M, Yoshitake Y, KouzakiM, Fukuoka H, and Fukunaga $T$ (2003) Strength training counteracts motor performance losses during bed rest. J Appl Physiol 95: 1485-1492

Tracy BL, Maluf KS, Stephenson JL, Hunter SK, Enoka RM (2005) Variability of motor unit discharge and force fluctuations across a range of muscle forces in older adults. Muscle Nerve 32: 533-540

Tracy BL (2007) Visuomotor contribution to force variability in the plantar flexor and dorsiflexor muscles. Hum Mov Sci 26: 796-807

Received: August 18, 2009

Accepted: January 6, 2010

Correspondence to: Kazushige Oshita, Graduate School of Human Development and Environment, Kobe University, 3-11

Tsurukabuto Nada-ku Kobe 657-8501, Japan

Phone: +81-078-803-7769

Fax: +81-078-803-7769

e-mail: 062d844d@stu.kobe-u.ac.jp 